\title{
SIMPLE AND ROBUST AIR GAP-BASED MEMS SWITCH TECHNOLOGY FOR RF-APPLICATIONS
}

\author{
P. Ekkels ${ }^{l, 2}$, X. Rottenberg ${ }^{l, 2}$, P. Czarnecki $^{l, 2}$, R. Puers ${ }^{2}$ and H.A.C. Tilmans ${ }^{l}$ \\ ${ }^{1}$ IMEC, Kapeldreef 75, 3001 Heverlee, Belgium \\ ${ }^{2}$ K.U.Leuven, Kasteelpark Arenberg 10, 3001 Heverlee, Belgium
}

\begin{abstract}
This paper presents a simple and robust process for fabrication of functional electrostatic RF-MEMS switching devices with lifetimes easily exceeding $10^{8}$ cycles with unipolar actuation at $100 \mathrm{~Hz}$. The device implements a switchable air gap capacitor and is therefore not limited in lifetime by dielectric charging as opposed to contact-type capacitive switches implementing high-k dielectrics. It is shown how these switched capacitors, even though having a capacitance ratio of only 2.8 , can still form adequate switching devices and RF-circuits by proper design and combining of these devices with high-Q inductors and transmission lines. The novelty of the proposed process is that it combines a sacrificial layer consisting of a single layer with a single dry etching step for the dimples which define the air gap in the down-state. This airgap is switched by electrostatic actuation of a thick electroplated Nickel bridge-structure. The device is realized in a 4-lithographic steps process with low complexity and high robustness.
\end{abstract}

\section{INTRODUCTION}

The main failure mechanisms in RF-MEMS switching technologies are related to either dielectric charging for capacitive switches or contact degradation for ohmic switches [1]. To overcome these problems an increasing demand on the packaging and of the process complexity can be observed. By removing the dielectric from the active area of capacitive switches, many of the original failure mechanisms are removed. However, substrate charging may still remain an issue, as demonstrated in [2] [3]. At the same time, removing the typically thin high-k dielectric from the capacitive device reduces the capacitance ratio, which is an important figure of merit of an RF-MEMS capacitive switch. Nevertheless, several groups have demonstrated capacitive switching devices without dielectric that show a good performance [4][5]. But, even though capacitance ratios in the order of 5 to 10 are achieved, the used processes are relatively complex, using multiple sacrificial layers or dimples that result from sacrificial layer planarization effects. Furthermore, through proper design, even low capacitance ratio technologies combined with high-Q inductors and transmission lines still offer the possibility to design adequate switching devices as well as RF-circuits. In this paper we present a simple process that allows the realization of reliable RF-MEMS switching devices with a high functionality and a long lifetime.

\section{BASIC CONCEPT}

The switchable capacitor we propose consists of an electrostatically actuated bridge structure that switches an air gap capacitor. The bridge consists of a thick membrane which is suspended by four relatively weak springs. Dimples on the bottom side of the bridge define the air gap in the down state (after pull-in) as illustrated in Figure

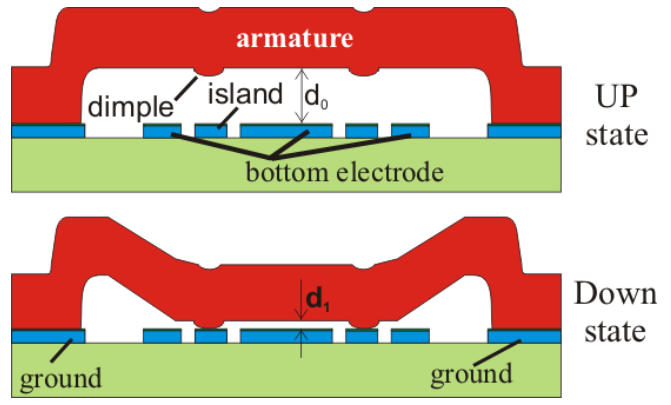

Figure 1: Sketch of the basic working principle of the switchable capacitor.

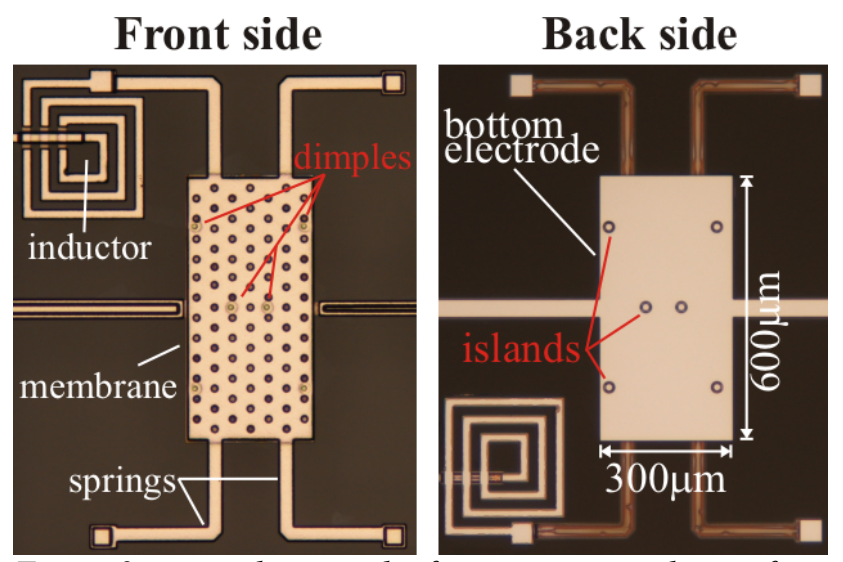

Figure 2: microphotograph of an air gap switch seen from front side and back sides.

1. The membrane has a much higher (bending) stiffness than the springs. This high stiffness together with a strategic placing of the dimples below the membrane prevents the membrane to collapse on the bottom electrode in the down-state. The 500nm high dimples land after pull-in on circular electrically isolated islands within the bottom electrode as shown in Figure 2. Since the capacitance of a parallel plate capacitor is directly related to the distance between the plates, the capacitance ratio $\mathrm{C}_{\mathrm{down}} / \mathrm{C}_{\mathrm{up}}$ is defined by the ratio of the gap height in the up-state $\left(\mathrm{d}_{0}\right)$ and in down-state $\left(\mathrm{d}_{1}\right)$. The capacitance ratio can be improved by either increasing $\mathrm{d}_{0}$, which would result in a higher pull-in voltage, or reducing $d_{1}$, by decreasing the height of the dimples. The scaling of both parameters is limited by the electric field in the air gap in the down state, which should remain below the breakdown field $\left(\mathrm{E}_{\text {breakdown }}\right)$ of the operating atmosphere:

$$
\frac{V_{\text {pull-in }}}{d_{1}}=\sqrt{\frac{8 k d_{0}^{3}}{27 \varepsilon_{0} A}} \cdot \frac{1}{d_{1}} \leq E_{\text {breakdown }}
$$

where $k$ the spring constant for the 4 springs combined, $A$ the area of the membrane and $\varepsilon_{0}$ the permittivity of free space. The breakdown field is strongly dependent on the operation atmosphere and the air gap. The presented structure has a pull-in voltage of around 50 Volt, which result in an electric field close to $10^{8} \mathrm{~V} / \mathrm{m}$ for a $500 \mathrm{~nm}$ gap. 


\section{FABRICATION PROCESS}

The process consists of 4 lithographic steps. Figure 3 shows how the process starts with patterning a $1 \mu \mathrm{m}$ thick aluminum alloy $\left(\mathrm{AlCu}_{0.5 \%}\right)$ bottom metallization on a $200 \mathrm{~mm}$ quartz wafer (1). On top of this layer a $3 \mu \mathrm{m}$ thick PW-1530 polyimide sacrificial layer is spin-coated and photolithographically patterned. After the $250^{\circ} \mathrm{C}$ crosslink bake, 500nm deep pits which define the dimples below the membrane are dry etched in the polyimide (2). Next a $10 \mu \mathrm{m}$ thick nickel film is electroplated through a photoresist mold (3). This layer serves both as the armature material and as signal line metallization as Figure 4 illustrates. Finally, the sacrificial layer is removed in an $\mathrm{O}_{2}$-plasma (4).

The dimples below the membrane land on islands fabricated in the same bottom metallization as the electrode. A good planarization of the slots between the islands and the electrodes in the bottom metallization is needed to guarantee that the polyimide has the same thickness above the islands and the electrode. This way only the depth of the etched pits will determine the downstate air gap in the final device as illustrated in Figure 5. By reducing the slot width to $5 \mu \mathrm{m}$ the polyimide film only follows the bottom metallization topology above the slot, but remains the same thickness above the islands and the electrode. This results in a rim around the dimple on the membrane backside as can be observed in Figure 6.

1)

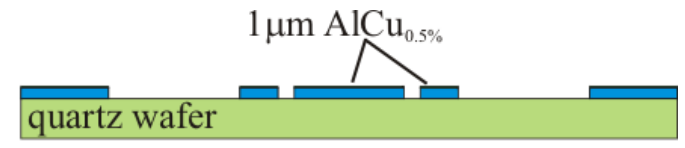

2)

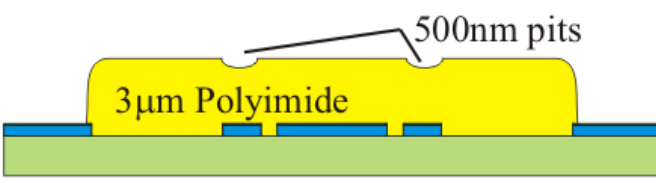

3)

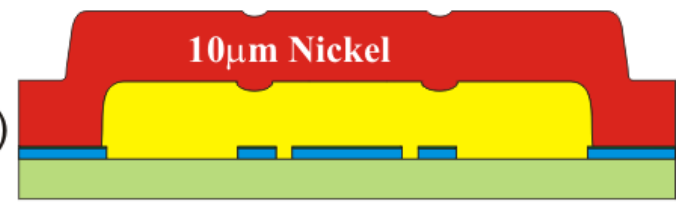

4)

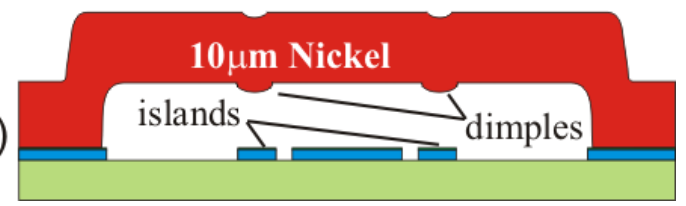

Figure 3: Sketch of fabrication process.

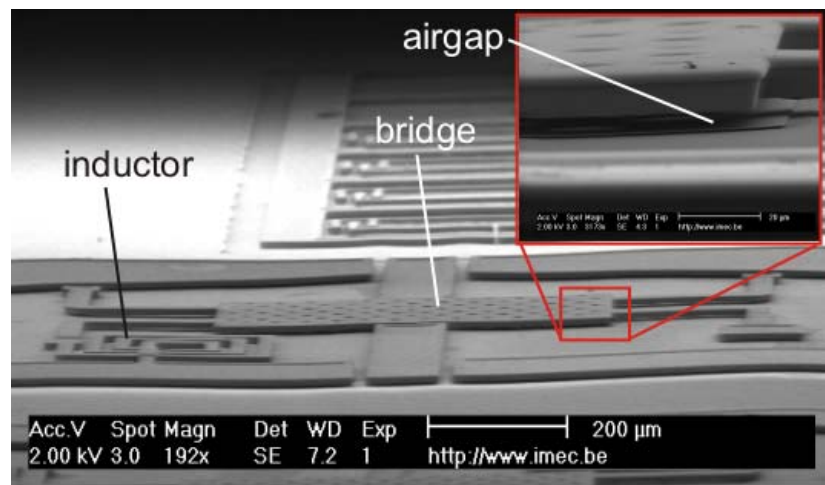

Figure 4: SEM image of topview of the device.

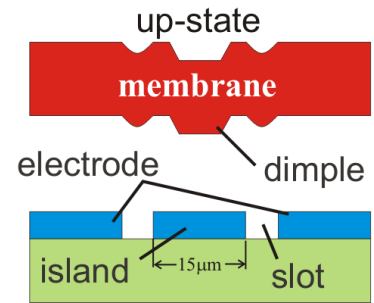

down-state

Figure 5: Sketch of membrane at the location of the dimples.

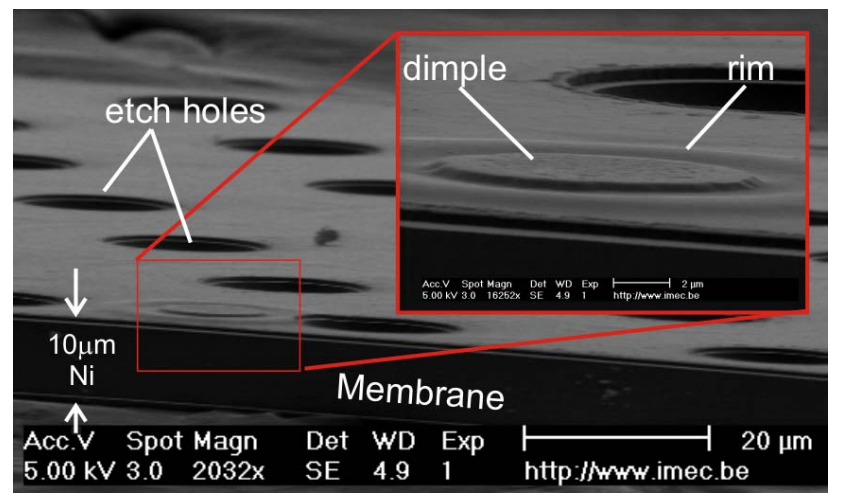

Figure 6: SEM image of the membrane backside.

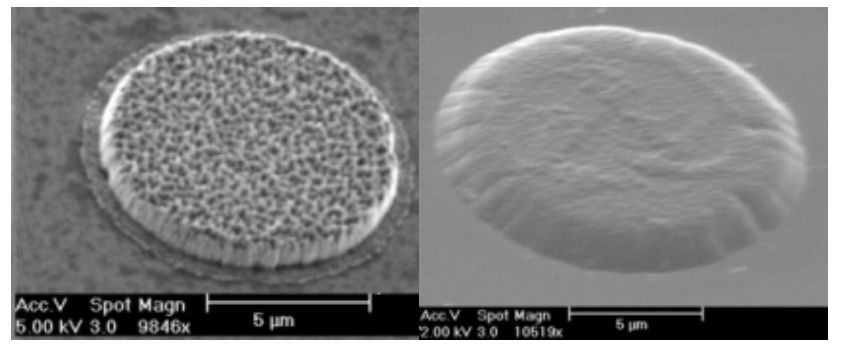

Figure 7: SEM picture of a Figure 8: SEM picture of a $1 \mu \mathrm{m}$ high dimple etched in $1 \mu \mathrm{m}$ high dimple etched in a parallel plate reactive ion a downstream resist asher etching system. system.

For the etching of the pits a comparison has been made between parallel plate reactive ion etching (RIE) and etching in a downflow reactor system. Tips that result from pit etching in the RIE system as shown in Figure 7, present a much higher roughness than tips resulting from pit etching in a Matrix Bobcat downflow reactor system as shown in Figure 8. The latter also have a more isotropic profile more favorable for step coverage by the seed layer. Since the etching of the pits is done through a hard mask with a small number of openings, the loading during the plasma etch is relatively low. This results in a high uniformity of the depth of the etched pits for both processes (etch-depth variation of $\sim 2 \%$ on $200 \mathrm{~mm}$ wafer). The armature is realized by electroplating nickel through a photoresist mold with a $\mathrm{Ti}(30 \mathrm{~nm}) / \mathrm{Cu}(500 \mathrm{~nm})$ seed layer. The relatively thick seed layer guarantees a good step coverage at the edges of the sacrificial layer which is important to achieve a uniform distribution of the plating current-density over the wafer. This uniformity is required for a high plating uniformity. A high density of nickel structures further helps to increase the plating uniformity. Nevertheless a variation in thickness of $3 \%$ within a structure and of $\sim 6-8 \%$ on a $200 \mathrm{~mm}$ wafer is observed. The stress of the nickel film after plating is $\sim 50 \mathrm{MPa}$ compressive, but after an anneal at $180^{\circ} \mathrm{C}$ the stress becomes 150-200MPa tensile. 


\section{MEASUREMENTS \& DISCUSSION}

For the realized devices, the stiffness of the $400 \mu \mathrm{m}$ long, $30 \mu \mathrm{m}$ wide springs by which the membranes are suspended is relatively low compared to the stiffness of the membrane itself. Nevertheless a voltage of $45 \mathrm{~V}$ is required for pull-in. The $\mathrm{C}-\mathrm{V}$ measurement, depicted in Figure 9, does not show the sharp pull-in as commonly observed with capacitive switches but show an initially more graduate change in capacitance. The low capacitance ratio of $1: 1.8$ is expected to result from parasitic capacitances and the measurement equipment.

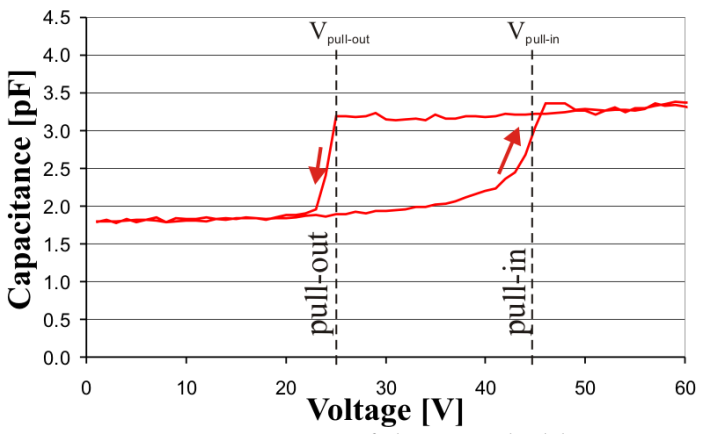

Figure 9: C-V measurement of the switchable capacitor.

The device with these basic characteristics enables the realization of several RF-circuits, two of which are presented in this paper. The first is the narrow band RFswitch shown in Figure 10 that functions as a tunable notch band filter while the second is the tunable stop band filter depicted in Figure 13. For the RF-switch the signal transmission in the up-state, characterized by the $S_{21}$ parameter, is optimized by matching the up-state capacitance of the switchable capacitor and the inductive

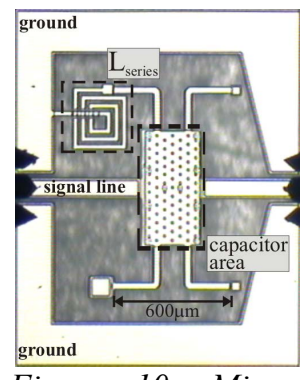

Figure 10: Microphotograph of a narrow band $R F$ switch.

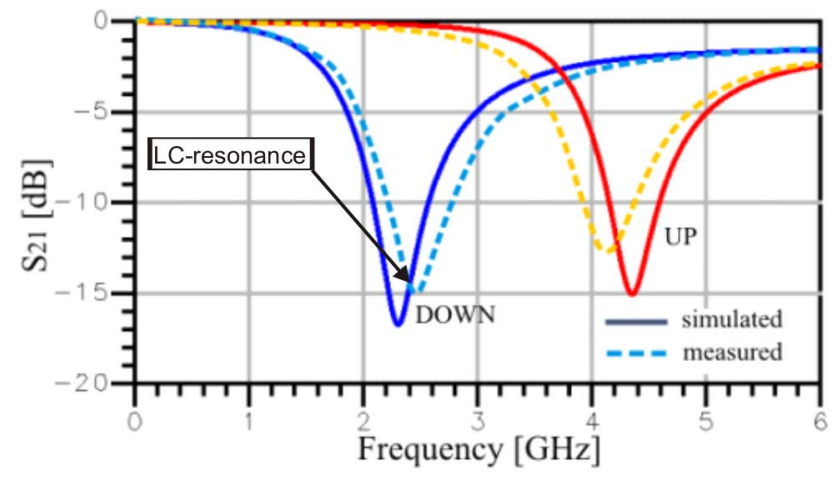

Figure 12: Simulation results and measured characteristics of the capacitive air gap RF-MEMS-switch of Figure 10 and 11, illustrating the narrow band behavior of the switch. feeding lines. In the down- state, $\mathrm{S}_{21}$ is strongly influenced by the resonance of the lumped LC-circuit between the MEMS and the ground that is created by adding series inductor $\mathrm{L}_{\text {series. }}$ This high-Q inductor is realized in the thick Ni layer and placed in the space created by the matched feeding lines. This way a compact low frequency LC tank is defined of which the circuit representation is shown in Figure 11. The device shows a shift in LCresonance from $4.2 \mathrm{GHz}$ in the UP-state to $2.5 \mathrm{GHz}$ in the DOWN-state as depicted in Figure 12. This results in a transmission loss of $0.3 \mathrm{~dB}$ and an isolation of $-15 \mathrm{~dB}$ at $2.5 \mathrm{GHz}$. The shift in resonance frequency matches a capacitance ratio of 2.8 , which is lower than the capacitance ratio of $\sim 6$ expected from the change in air gap. The cause for this deviation is currently being investigated. We can further improve the switching characteristics of this circuit by implementing a more complex 2-stage circuit shown in Figure 13 and Figure 14. Here two RF switches in shunt configuration are coupled by an inductor $\left(\mathrm{L}_{\text {couple }}\right)$. This way a tunable stopband filter is defined which has a steep cut off and a wide rejection

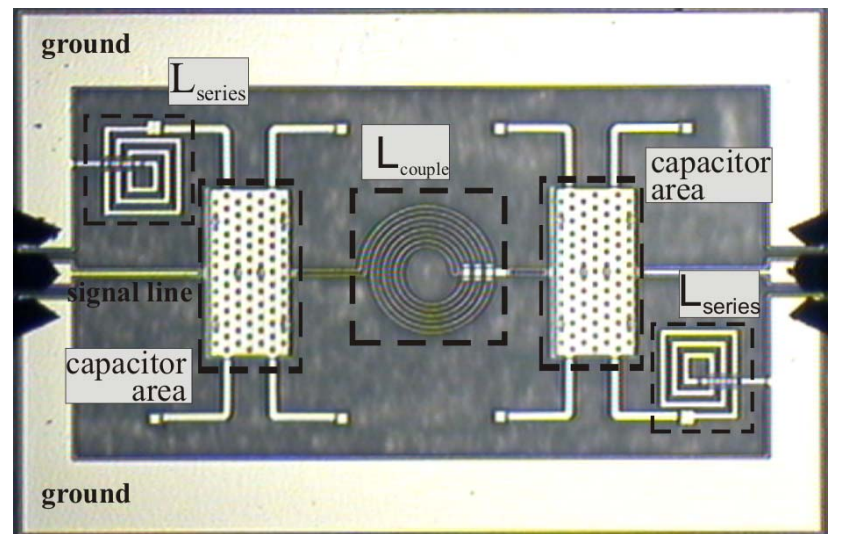

Figure 13: Microphotograph of a tunable stopband filter.

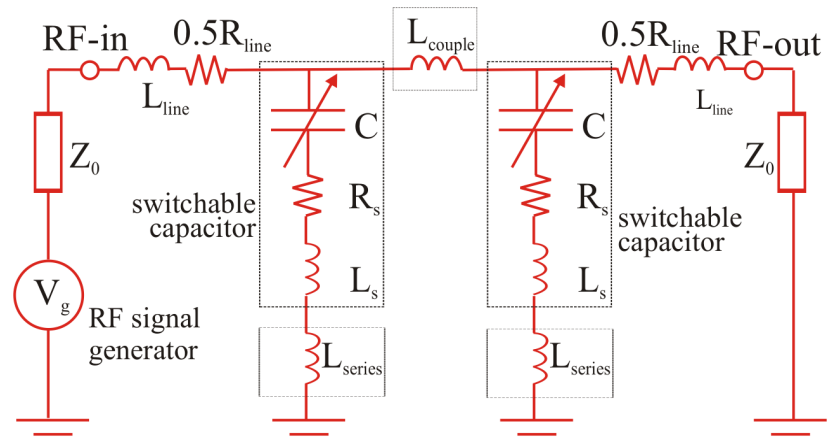

Figure 14: Circuit representation of the tunable stopband filter.

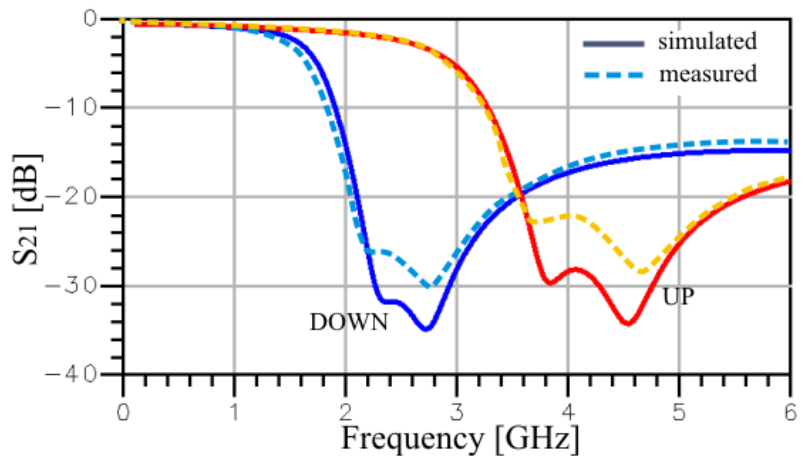

Figure 15: Simulation results and measured characteristics of the tunable stopband filter of Figure 12. 
band as shown in Figure 15. This device shows a shift in LC-resonance from $3.8 \mathrm{GHz}$ in the UP-state to $2.2 \mathrm{GHz}$ in the DOWN-state, and a transmission loss of $1.4 \mathrm{~dB}$ with an isolation of $-26 \mathrm{~dB}$ at $2.2 \mathrm{GHz}$. Improvements of the design should significantly reduce the transmission loss. The main cause of failure for capacitive switching devices is stiction due to interposer dielectric charging of the high- $\mathrm{k}$ dielectric between the bridge and bottom electrode. Since there is no interposer dielectric in the device presented here, the only remaining source of charging is substrate charging [2]. Lifetime measurements with $50 \mathrm{~V}$ unipolar actuation, $100 \mathrm{~Hz}$ and $50 \%$ duty cycle in $\mathrm{N}_{2}$ as depicted in Figure 16 showed no failure during $10^{8}$ cycles, after which the measurement was stopped. The only significant shifts in the up and down capacitance are the result of thermal drift in the equipment during the measurement. In order to obtain more information on the charging behavior both the pull-in and pull-out voltage were monitored during the lifetime measurements as shown in Figure 17. Only a mild shift of the overall $\mathrm{C}-\mathrm{V}$ characteristics is visible while both pull-in and pull-out window clearly narrow, testifying of non-uniform charging [6] [7]. The narrowing is most significant for the pull-out window is defined as $\mathrm{d} \Delta \mathrm{V}_{\mathrm{po}}=\left(\mathrm{V}_{\mathrm{po}}+\right)-\left(\mathrm{V}_{\mathrm{po}}-\right)$. Figure 18 shows how the

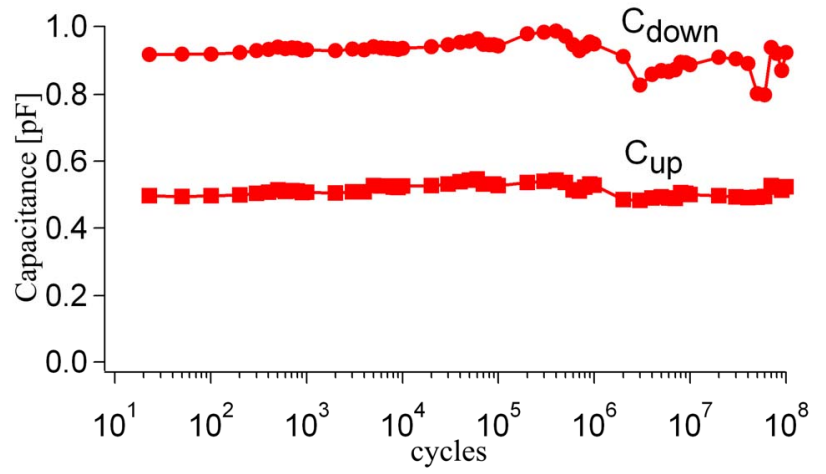

Figure 16: Lifetime of an air gap switch using $50 \mathrm{~V}$ unipolar actuation, $100 \mathrm{~Hz}$ and $50 \%$ duty cycle in $\mathrm{N}_{2}$ environment.

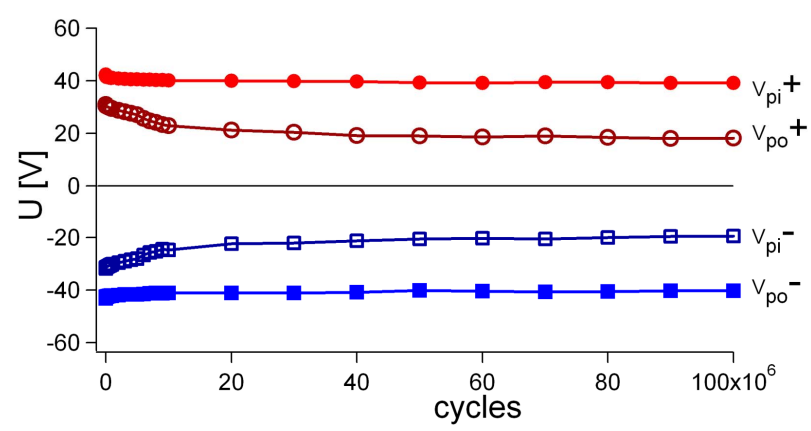

Figure 17: Monitored pull-in and pull-out voltages of air gap switch using 50V unipolar actuation, $100 \mathrm{~Hz}$ and $50 \%$ duty cycle in $\mathrm{N}_{2}$ environment.

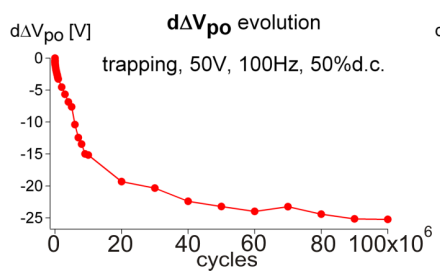

Figure 18: Narrowing of the pull-out window during switching. narrowing stabilizes after $\sim 4 \cdot 10^{7}$ cycles due to saturation of the substrate charges. It is observed that after the loading ended the charges quickly disappear, which results in a widening of the pull-out window as shown by the de-trapping experiment of Figure 19. The lifetime of these devices is thus not limited by the substrate charging and significant longer lifetimes can be realized.

\section{CONCLUSION}

In this paper we have presented a simple and robust technology for realization of RF-MEMS circuits with a high functionality and long lifetimes The technology consists of a 4 lithographic steps process which is used to create a MEMS switchable capacitor. It consists of a $10 \mu \mathrm{m}$ thick Ni membrane which is used to switch the height of an air gap dielectric. Even though the resulting capacitance ratio of of 2.8 is relatively small the thick metallization can be used to create compact low frequency LC-tanks which allows the creation of several interesting RF-circuits. Due to the absence of an interposer dielectric the only source of charging is substrate charging of the quartz substrate. Stable lifetimes of at least $10^{8}$ cycles with unipolar actuation have been demonstrated but a much longer lifetime is expected.

\section{REFERENCES}

[1] G. M. Rebeiz, "RF MEMS in theory, design and technology", Hoboken, NJ, USA, Wiley, 2003.

[2] P. Czarnecki, X. Rottenberg, P. Soussan, P. Ekkels, P. Muller, P. Nolmans, W. De Raedt, H. A. C. Tilmans, R. Puers, L. Marchand and I. De Wolf, "Influence of the substrate on the lifetime of capacitive RF MEMS switches", proc. of MEMS 2008, pp 172-175, 2008.

[3] X. Rottenberg, P. Nolmans, P. Ekkels, P. Czarnecki, R. P. Mertens, B. Nauwelaers, I. De Wolf, W. De Raedt and H. A. C. Tilmans, "Electrostatic fringing-field actuator (EFFA): application towards a low-complexity RF-MEMS technology, proc. of MEMS 2007, pp 203-206, 2007.

[4] Qin Shen, N. S. Barker, "Distributed MEMS Tunable Matching Network Using Minimal-Contact RF-MEMS Varactors", IEEE transactions on microwave theory and techniques, Vol 54, no 6, pp2646-2658, 2006.

[5] P. Blondy, A. Crunteanu, C. Champeaux, A. Catherinot, P. Tristant, O. Vendier, J. L. Caw and L. Marchand, "Dielectric Less Capacitive MEMS Switches", MTT-S Microwave Symposium Digest, Vol. 2, june 2004, pp. 573576.

[6] X. Rottenberg, I. De Wolf, B. K. J. C. Nauwelaers, W. De Raedt and H. A. C. Tilmans, "Analytical model of the DC actuation of electrostatic MEMS devices with distributed dielectric charging and non-planar electrodes", JMEMS, vol. 16(5), pp. 1243-1253, October 2007.

[7] P. Czarnecki, X. Rottenberg, P. Soussan, P. Nolmans, P. Ekkels, P. Muller, H. A. C. Tilmans, W. De Raedt, R. Puers, L. Marchand, I. De Wolf, "Experimental evidence of non-uniform dielectric charging in capacitive RF-MEMS switches", 18th Workshop on MicroMechanics Europe, pp 227-230, 2007. 